\title{
A Hybrid Model for Short-Term Wind Power Forecasting Based on MIV, Tversky Model and GA-BP Neural Network
}

\author{
Jie Zeng ${ }^{1}$, Chi Zhang ${ }^{1}$, Ning $X_{i e}{ }^{1}$, Ping Yang ${ }^{2,3}$, Chenyu $X^{2,3}$ and Zhen Zhang ${ }^{4}$ \\ ${ }^{1}$ Electric Power Research Institute, Guangdong Power Grid Corporation, 510640, Guangzhou, China \\ ${ }^{2}$ School of Electric Power, South China University of Technology, 510640, Guangzhou, China \\ ${ }^{3}$ Guangdong Key Laboratory of Clean Energy Technology, South China University of Technology, 511458, Guangzhou, China \\ ${ }^{4}$ Guangdong Intework Energy Technology Co., Ltd, 511458, Guangzhou, China
}

\begin{abstract}
Wind power forecasting, which is necessary for wind farm, is significant to the dispatch of power grid since the characteristics of wind change intermittently. In this paper, a hybrid model for short-term wind power forecasting based on MIV, Tversky model and GA-BP neural network is formulated. The Mean Impact Value (MIV) method is used to monitor the input variable of BP neural network which will simplify the neural network model and reduce the training time. And the Tversky model is used for cluster analysis, which keeps watch over the similar training set of BP neural network. In addition, the Genetic Algorithm (GA) is used to optimize the initial weights and thresholds of BP neural network to achieve the global optimization. Simulation results show that the method combined with MIV, Tversky and GA-BP can improve the accuracy of short-term wind power forecasting.
\end{abstract}

\section{Introduction}

The output power of the wind farm fluctuate frequently, bringing great difference to grid-connection [1]. In order to facilitate the power grid dispatching, it is critical to forecast short-term wind power accurately. At present, there are two main methods to forecast short-term wind power, physical method and statistical method. There are two ways to achieve the physical method [2], [3]. One way is to fit the parameters of the wind speed and the direction from the numerical weather forecast and transfer it to wind speed in each wind turbine in order to get the wind power of each wind turbine and then calculate wind power of wind farm [4]. Another way is to establish a high-accuracy three-dimensional atmospheric physical model and calculate the wind speed and wind direction of each wind turbine based on the boundary conditions of the wind farm, which can finally be transferred to wind power of wind farm [5]. The main methods of statistical prediction are time series method [6], neural network [7], Support Vector Machine (SVM) [8], etc. The time series method establishes a time series model based on a large number of historical data and forecasts wind power with the time series model. This method is simple, but it is only suitable for the ultrashort-term wind power forecasting because the forecasting accuracy is lower when ahead time is too high. Neural network method is based on the human brain information mechanism. It has the advantages of fast calculating, good robustness and good learning ability. But there are also disadvantages of neural network. The training time of neural network is long. Moreover, neural network has high demand for data and easily fall into local optimum [9]. The support vector machine method transfers the non-linearly unseparable samples in low dimensional space into linearly separable one in high dimensional space via kernel function, which can greatly avoid local optimum. But this method don't make enough analysis of input variables so the accuracy is not high. In order to improve the accuracy of the forecasting of wind power, it is important to make pretreatment so that the data can better meet the needs of model.

In this paper, we argue a short-term wind power forecasting method based on MIV, Tversky model and GA-BP neural network. First, we calculate the Mean Impact Value (MIV) of each input variable based on BP neural network [10], [11]. Then, the variables will be sorted in order to find the key input items. The Tversky model is used to cluster analysis. Finally, the Genetic Algorithm (GA) optimizes the initial weights and thresholds of Back Propagation (BP) neural network to achieve the global optimization [12]. This method can optimize the input variables to improve the accuracy of short-term wind power forecasting.

\section{Optimized feature vector based on MIV}

In wind power forecasting, the input variables include three layers of WRF generally. In each layer, there are 7 input variables include wind speed of longitude direction, wind speed of latitude direction, vertical wind speed, air pressure, geopotential height, temperature and humidity. The height of the general wind turbine is close to second 
layer, so we use the variables in second layer to calculate. The process of calculating Mean Impact Value (MIV) is as follows.

Firstly, we use the training set to get the training model, increasing and reducing each training input variable values by $10 \%$ and respectively constitute two new sample set. Secondly, we take the two new sample set as the simulation sample set and use the trained model to calculate the output value of the samples and the subtraction, which is the Impact Value (IV) of influence of the changing argument, and the mean value of the IV of each variable is Mean Impact Value (MIV). Finally, the MIV value of each variable should be arranged to find the relative influence of each variable, from which we can select the input variables of neural network out.

\section{Samper cluster analysis based on Tversky model}

In this paper, a similar sample clustering method is used to pretreat the data samples. Write forecasting samples and the training samples into vector and then filter out the training data which is similar to forecasting samples by calculating vector similarity. Then use the pretreated data as train sample of neural network and establish model for forecasting.

The method to measure vector similarity can be divided into 2 types, Geometric Model and Feature Contrast Model. In geometric model, each point represents a sample, and the distance between the points similarity represents the similarity of the vector. The distance of each dimension in the geometric model is symmetrical, however, that in wind power forecasting is not. So it's not suitable to use geometric model in shortterm wind power forecasting. In feature contrast model, it is common to use Tversky model. Using Tversky model means assuming that the similarity of vector satisfies three characteristics, which is the independence, monotonicity and matching. Based on the assumption, the Tversky model gives the following theorem:

Assuming that $s$ is a similarity function, which satisfies the three characteristics of the assumption. Then we can find a similarity function Sim, a non-negative function $f$ and constant $\theta, \alpha, \beta$. For all objects $a, b, c, d$, we have:

$$
\begin{aligned}
& \operatorname{Sim}(a, b) \geq \operatorname{Sim}(c, d) \Leftrightarrow s(a, b) \geq s(c, d) \quad \text { and } \\
& \operatorname{Sim}(a, b)=\theta f(A \cap B)-\alpha f(A-B)-\beta f(B-A)
\end{aligned}
$$

where $A$ and $B$ are sets of vector $a$ and $b, \alpha$ and $\beta$ describe the inconsistent feature vector.

In this paper, we use the cosine function to compare the similarity between two vectors. Assuming that $S, S$, are feature vector in the sample set. The method to calculate similarity using cosine function can be expressed as follows:

$$
\operatorname{Sim}_{c f}\left(S, S^{\prime}\right)=\frac{\sum_{i=1}^{n} S_{i} * S_{i}^{\prime}}{\sqrt{\sum_{i=1}^{n} S_{i}^{2}} * \sqrt{\sum_{i=1}^{n} S_{i}^{\prime 2}}}
$$

By comparing the similarity of vector with cosine function, we can pick out the samples which are similar to forecasting set. The process of selecting samples is shown as follows:

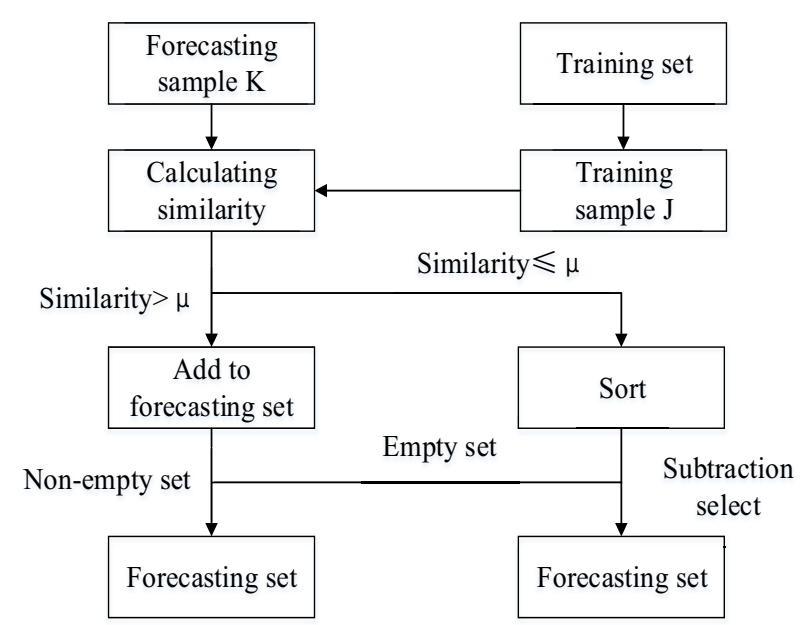

Figure 1. The process of selecting samples.

\section{Genetic Algorithm (GA) optimizes initial features of neural network}

\subsection{Steps of genetic algorithm}

In this paper, Genetic Algorithms (GA) is used to optimize initial weights and thresholds of Back Propagation (BP) neural network. Commonly BP neural network has random initial values. With the help of GA, BP neural network can be forecasted better.

Genetic algorithm is an optimization algorithm, which can be realized by simulating the natural genetic law. According to the natural genetic law, GA takes each solution as an individual which is possibly the optimal solution. Similar to the theory of evolution, GA set up a fitness function to distinguish the individual fitting well. After selection, crossover and mutation, we will save the individuals who fit well and eliminate those who fit bad in order to ensure that the new generation fit better than previous generation. Finally we can obtain the optimal solution.

Genetic algorithm includes the following steps.

\subsubsection{Initialize population}

Each individual should be a possible solution. In this paper, the individual should include the weights and thresholds of the BP neural network. In the case of BP neural network structure determined, each individual can determine the weights and thresholds of the neural network. So we can use BP neural network to judge whether this solution is the optimal solution.

\subsubsection{Calculating fitness function}

Calculate the fitness of each individual by fitness function. According to each individual we can determine the weights and thresholds of neural network. By using 
BP neural network, we can obtain the predicted wind power output. It is obvious that the smaller difference between the actual output and the predicted output, the higher fitness of the individual. Therefore, we can define the individual's fitness function as the error between the predicted output and the actual output, which can be expressed by the following formula.

$$
f=\frac{1}{\sum_{i=1}^{n} a b s\left(y_{i}-o_{i}\right)}
$$

where $n$ is the nodes number of output layer of neural network, $y_{i}$ is predicted output of node $i$ and $\underline{o}_{i}$ is actual output of node $i$.

As can be seen from the above, the smaller error of the individual's fitness is, the larger fitness function is. The Individual with great fitness pass information to the next generation ensuring the generation better than the former.

\subsubsection{Operation of selection, crossover and mutation}

In order to enrich the kinds of operator, we need to use three operations such as selection, crossover and mutation.

The operator selected is based on fitness strategy which contains the methods of roulette and championship commonly. We chose the method of roulette in this paper. The probability of selecting an individual is expressed as follows:

$$
p_{i}=\frac{f(i)}{\sum_{i=1}^{N} f(i)}
$$

where $\mathrm{N}$ represents the number of operators. The probability of each operator is proportional to the fitness. So the greater fitness of the operator is, the higher probability to be chosen.

Since each operator is showed by encoding, the method of crossover can be used to deal with the cross operation of each operator. To explain the process of cross algorithm, assume that there are two chromosomes called $m$ and $n$, the crossover operation between the two chromosomes at position $\mathrm{K}$ is expressed as follows:

$$
\left\{\begin{array}{l}
a_{m k}=a_{m k}(1+b)-a_{n k} b \\
a_{n k}=a_{n k}(1-b)+a_{m k} b
\end{array}\right.
$$

where $b$ is a random number in the interval $[0,1]$.

The purpose of mutation operation is to increase the diversity of the operator. By mutating the operator of a certain position, the type of operator will increase. Hypothesizing changing the gene $a_{i j}$ of $j$ in the individual $I$, the operation method is shown as follows:

$$
\begin{gathered}
a_{i j}=\left\{\begin{array}{c}
a_{i j}+\left(a_{i j}-a_{\max }\right) * f(g), r \geq 0.5 \\
a_{i j}-\left(a_{\min }-a_{i j}\right) * f(g), r<0.5
\end{array}\right. \\
f(g)=r_{2}\left(1-\frac{g}{G_{\max }}\right)
\end{gathered}
$$

where $a_{\max }$ and $a_{\min }$ represent the maximum boundary and minimum boundary of gene $a_{i j}, r_{2}$ is a random number in the interval $[0,1], g$ is the present number of iteration, $G_{\max }$ is the maximum number of evolution.

\subsection{The process of GA-BP neutral network}

The process of GA-BP neutral network is shown as follows.

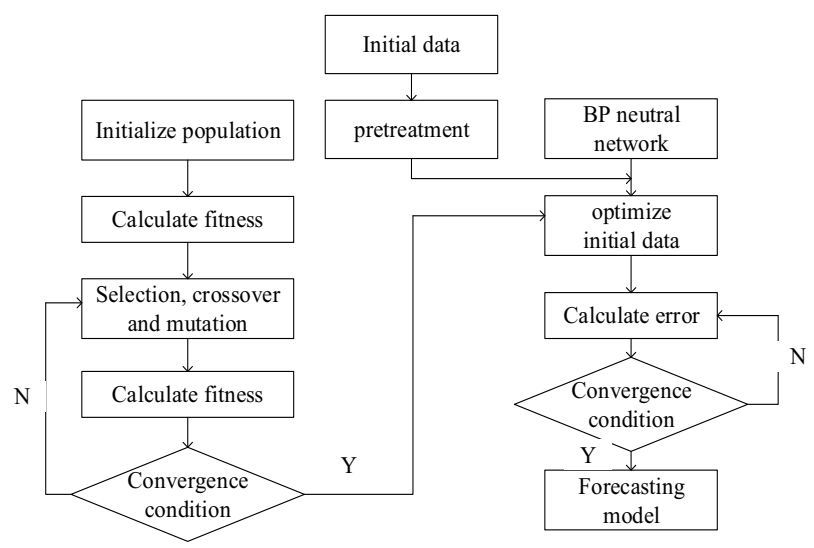

Figure 2. The process of GA-BP neutral network.

From the graph shown above, we can see that the genetic algorithm optimize the weights and thresholds of BP neural network, which is used to establish forecasting model of wind farm. Genetic algorithm can improve the accuracy of forecasting model by optimizing the initial weights and thresholds of BP neutral network.

\section{Simulation verification}

\subsection{Initial data}

In this case, the mode of numerical weather forecast data is WRF mode and the actual output data of wind farm is provided by the Power Grid Corp. The selected data is active power of high voltage side in the No. 1 main transformer, the time interval of data is 15 minutes. The observed data vary from 00:00:00, 2013-01-01 to 23:45:00, 2013-07-31. The latitude and longitude of NWP points is shown as follows,

Table 1. Latitude and longitude of NWP points.

\begin{tabular}{cccc}
\hline longitude & 108.42 & 108.39 & 108.37 \\
\hline Latitude & 18.79 & 19.02 & 19.25 \\
\hline
\end{tabular}

The time interval of NWP data is 15 minutes, the distance between two adjacent points is $27 \mathrm{~km}$. In this wind farm, there are 24 wind turbines, whose rated capacity are all $1500 \mathrm{~kW}$ and rated wind speed $12 \mathrm{~m} / \mathrm{s}$. The height of wind turbines is $70 \mathrm{~m}$.

\subsection{Results analysis}

First, we forecast wind power output without cluster analysis and directly use sample set for training. In this way, we obtain the forecasting wind power output curve. Then we use the method this paper explains to forecast wind power output and obtain the forecasting wind power curve. Finally, comparing with two output curves above and actual output curve we can obtain the result shown as follows. 


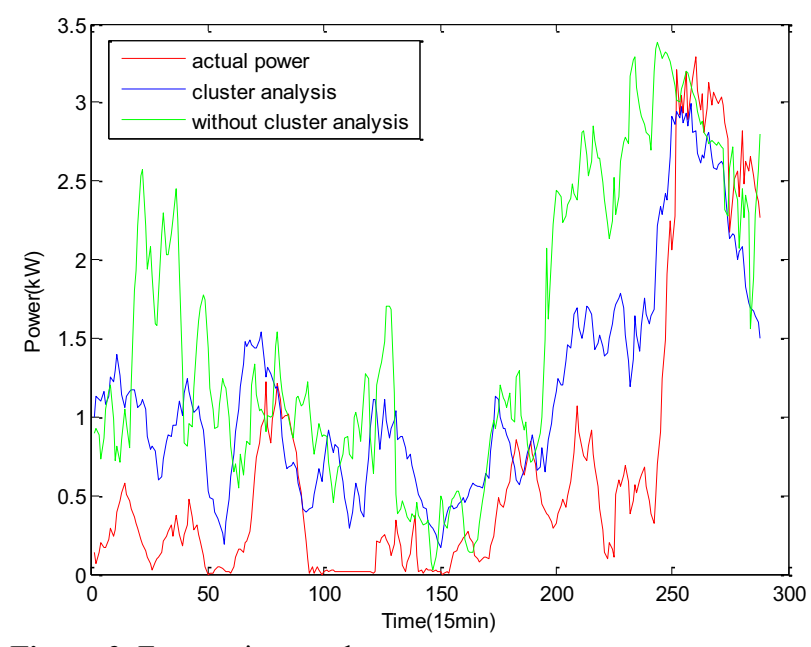

Figure 3. Forecasting results.

In the figure above, the red curve is actual wind power output curve of the wind farm, the blue curve is forecasting wind power output curve with the paper's explaining method and the green one is forecasting wind power output curve without cluster analysis. From the figure above, it is obvious that the forecasting method this paper explains can better forecast wind power output, for it can choosing the sample with higher similarity as the input parameter of BP neural network. Thus the neural network can be better trained and get more accurate results.

The forecasting error curves of forecasting method with cluster analysis and the method without cluster analysis are shown as follows.

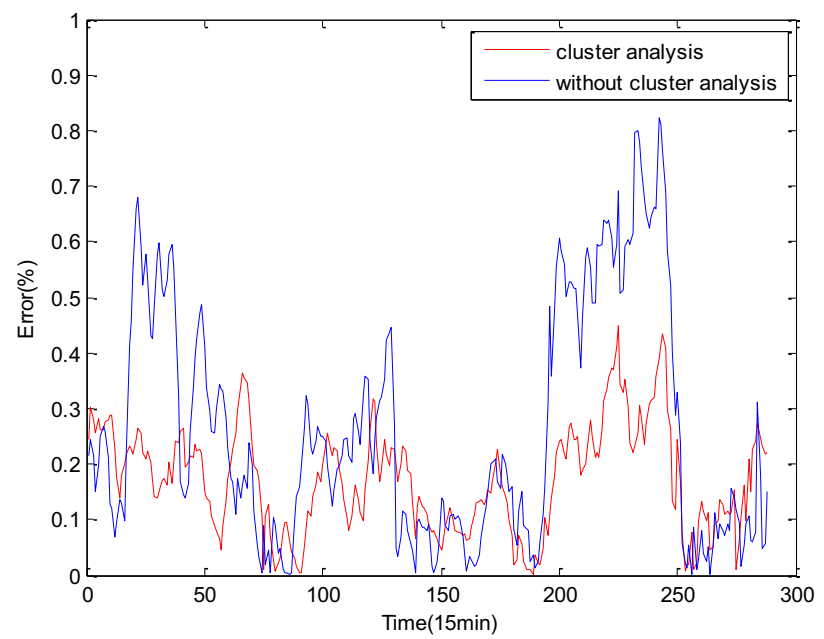

Figure 4. Forecasting error curves.

The forecasting root-mean-square errors (RMSE) of the two methods above are shown as follows.

Table 2. RMSE of the two methods.

\begin{tabular}{cc}
\hline Method of this paper & $18.6 \%$ \\
\hline Method without cluster analysis & $32.4 \%$ \\
\hline
\end{tabular}

The statistical forecasting model is suitable for the wind farm with a large amount of historical data. According to the forecasting result, the forecasting accuracy is obviously improved by Optimized the initial data.

\section{Conclusions}

In this paper, a BP neural network algorithm with MIV and Tversky model monitoring input variable and Genetic Algorithms optimizing initial the weights is presented. The BP neural network is used to calculate the Mean Impact Value (MIV) of each input variable so that the most useful data can be selected for training according to MIV. Then, for filtering out the training data which is similar to forecasting samples, the way of cluster analysis is put forward. At last, to optimize the initial weights and thresholds of BP neural network, GA-BP neutral network is used to establish forecasting model and obtain the forecasting wind power output value. From the case shown above, it is concluded that the algorithm formulated can greatly enhance the accuracy of shortterm wind power forecasting with shorter time.

\section{Acknowledgment}

This work was supported by Guangdong Strategic Emerging Industry Core Technology Research Projects (2012A032300013); National Science and Technology Support Program (2015BAA06B02).

\section{References}

1. M. A. Islam, et al., Global renewable energy-based electricity generation and smart grid system for energy security, Scientific World Journal, 2014:197136, (2014)

2. E. Pelikán, et al., Wind power forecasting by an empirical model using NWP outputs, in Environment and Electrical Engineering (EEEIC), 2010 9th International Conference on, IEEE: Prague, Czech Republic, (2010) 45

3. S. Al-Yahyai, A. Gastli, Y. Charabi, Probabilistic wind speed forecast for wind power prediction using pseudo ensemble approach, in Power and Energy (PECon), 2012 IEEE International Conference on, IEEE: Kota Kinabalu, (2012) 127

4. S. Buhan, I. Cadirci, Multi-Stage Wind-Electric Power Forecast by Using a Combination of Advanced Statistical Methods. Industrial Informatics, IEEE Transactions on. (99):1, (2015)

5. N. O. Jensen. A note on wind generator interaction, (1983)

6. J. Taylor, P. McSharry, Short-term load forecasting methods: Anevaluation based on European data, IEEE Trans. Power Syst., 22(4):2213-2219, (Nov. 2007)

7. H. Quan, D. Srinivasan, A. Khosravi, Short-term load and wind power forecasting using neural network-based prediction intervals. IEEE Trans. Neural. Netw. Learn. Syst., 25(2):303-15, (2014)

8. J. Wen, et al., Short-term wind power forecasting based on lifting wavelet transform and SVM, in Power Engineering and Automation Conference (PEAM), 2012 IEEE, IEEE: Wuhan, (2012) 1

9. H. Quan, D. Srinivasan, A. Khosravi, Incorporating Wind Power Forecast Uncertainties Into Stochastic 
Unit Commitment Using Neural Network-Based Prediction Intervals, IEEE Trans. Neural. Netw. Learn. Syst., (2014)

10. Y. Bashon, D. Neagu, M. J. Ridley, Fuzzy settheoretical approach for comparing objects with fuzzy attributes, in Intelligent Systems Design and Applications (ISDA), 2011 11th International Conference on, IEEE: Cordoba, (2011) 754
11. A. Tversky, Features of Similarity, Psychological Review, 84(4):327-352, (1977)

12. Y. Wang, S. Wang, N. Zhang, A novel wind speed forecasting method based on ensemble empirical mode decomposition and GA-BP neural network, in Power and Energy Society General Meeting (PES), 2013 IEEE, IEEE: Vancouver, BC, (2013) 1 